\title{
Energy Efficient MAC Protocol in Vehicular Networks
}

\author{
Swathy S \\ PG Scholar, M.E.C.S \\ Hindusthan College of \\ Engineering and Technology \\ Coimbatore, India
}

\author{
Ashok Kumar S \\ Assistant Professor, ECE \\ Hindusthan College of \\ Engineering and Technology \\ Coimbatore, India.
}

\begin{abstract}
In VANETs the trade off between energy efficiency and throughput is one of the main constraints since the medium is time varying wireless channel. In this work we introduce fragmented transmission scheme in to VANETs that improve the energy efficiency pursuing high throughput. In existing systems contention window and back off stage is adapted according to mobility of vehicular node. Along with impact of mobility current channel state also considered in this paper to make the system energy efficient. The simulation results show that the proposed scheme improves throughput making the system energy efficient.
\end{abstract}

\section{General Terms}

Markov model, Access point (AP)

\section{Keywords}

Vehicular Ad-hoc networks (VANETs), Distributed Coordination function (DCF), Energy Efficiency

\section{INTRODUCTION}

A Vehicular Ad-Hoc Network or VANET allow integration of vehicles and Intelligent Transportation Systems. A Vehicular Ad-Hoc Network or VANET is a technology that uses moving vehicles as nodes in a network to create a mobile network. VANET turns every participating vehicle into a wireless router or node allowing vehicles to connect and in turn, create a network with a wide range. As vehicles fall out of the signal range and drop out of the network other vehicles can join in, connecting vehicles to one another so that a mobile Internet is created. It is estimated that the first systems that integrate this technology are police and fire vehicles to communicate with each other for safety purposes. Driving is the process of changing the location constantly. This means a constant demand for information on the current location and specifically for data on the surrounding traffic, routes and much more. This information can be grouped together in several categories.

Vehicular traffic simulations can coarsely be classified into microscopic and macroscopic approaches. When following a macroscopic approach one focuses on system parameters like traffic density (number of vehicles per kilometer per lane) or traffic flow (number of vehicles per hour crossing an intersection) in order to compute a road's capacity or the distribution of traffic in a road net.. In contrast with a microscopic approach the movement of each individual vehicle is determined. In order to generate vehicle movement patterns for ad hoc routing experiments one clearly has to follow a microscopic approach because the position of each individual vehicle is needed. Nevertheless one also has to take care that a microscopic simulation does not result in unrealistic macroscopic effects.

\section{RELATED WORK}

In [3] using dynamic tuning of IEEE 802.11 theoretical throughput value can be achieved by tuning each station. Due to network congestion collision may occur in this case. Link throughput is maximized using context-aware rate selection in [7],but robustness to errors in context information is one of the major drawback.

The performance of VANETs in high mobility scenario is already discussed. The impact of mobility on throughput was the main constraint in VANETs.So to avoid the effect of mobility two enhancements done in [9](i)according to node velocity and network size $\mathrm{CW}_{\min }$ should adapt.(ii)To reduce impact of movement of vehicles in throughput the maximum back off stage is kept small.

Even though the impact of mobility on throughput is reduced considerably, the transmission is not much efficient in wireless medium. There are different factors which affect the wireless channel conditions. Due to fading and interference wireless channel is not stable.

\section{METHODOLOGY}

The system model we are considering is similar to that of [9]. Markov model is considered for the traffic environment. Road is divided in to seven lanes and protocol enhancement is applied to reduce impact of mobility. The transmission scheme according to the current channel state is applied to the system. Here we are considering fragmented transmission scheme as in [13].In FT, the current channel state is considered by measuring the received SNR value. Two threshold values are setup for finding out the channel state; Fragment threshold and Transmit threshold. If the received SNR is greater than fragment threshold the channel state is good and the full frame can be transmitted. If it is less than transmit threshold the channel state is considered to be bad and the transmission is deferred. For the case of SNR value in between these two thresholds, the channel state is medium. So full frame is divided in to two fragments and transmitted one by one. By FT the unsuccessful transmission can be avoided thereby reducing the frame error rate. Thus the tradeoff between throughput and energy efficiency is balanced.

The system model for the proposed scheme is given in fig.1.Here we are considering Markov model in which the road is divided in to seven lanes and access points (AP) at road side. First to reduce the impact of mobility the $\mathrm{CW}_{\text {min }}$ adapted according to velocity and back off stage is kept small. Then for the node transmitting first, the received SNR is measured by the AP and is compared with the thresholds. 
Thus the channel state is decided and is informed to the sending node with the return frame. According to the information in the return frame the sending node either send the full frame or fragmented frame or defer transmission.
Then the throughput value is measured and compared with the throughput value before applying the FT. The energy efficiency value is also plotted.

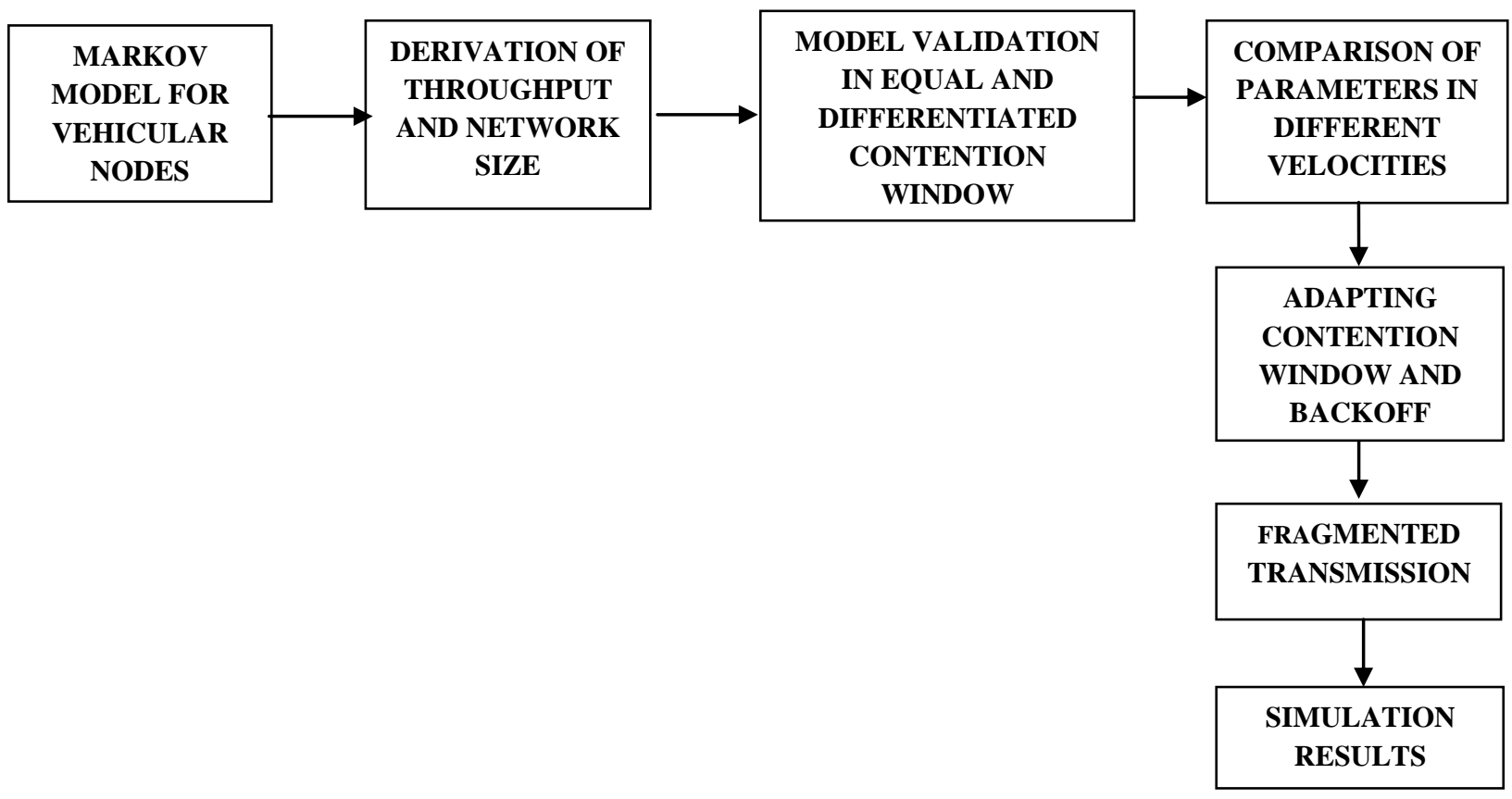

Fig 1: Flow Diagram

\section{SIMULATION SETUP}

For evaluation purpose a traffic environment is simulated in ns2, in which APs are deployed along the road and the vehicles passing through compete for communications using IEEE 802.11b.The whole road segment is divided into different lanes within the radio coverage of AP. A total of 125 nodes are considered for simulation. In 125 nodes 13 nodes are taken as mobile nodes in the traffic environment.

\section{RESULTS AND DISCUSSION}

The performance of developed system is considered in different velocities. Different parameters like system throughput, packet delivery ratio, collision probability and energy remaining are plotted.

In Fig 2 the system throughput, for two cases are compared. The system throughput after applying FT is high compared to the other. This is due to the fact that the unsuccessful transmission is mitigated and also the impact of mobility is reduced.

Packet delivery ratio is the number of packets received out of the packets sent in percent. After applying the FT the packet delivery ratio is increased marginally in Fig 3 .

Collision probability is the probability of collision during transmission. By considering the current channel state the collision can be avoided in FT.

Energy consumption is the amount of energy utilized in this scheme. After applying FT the total energy consumed is comparatively less. This lead to an efficient MAC protocol in VANETS. 


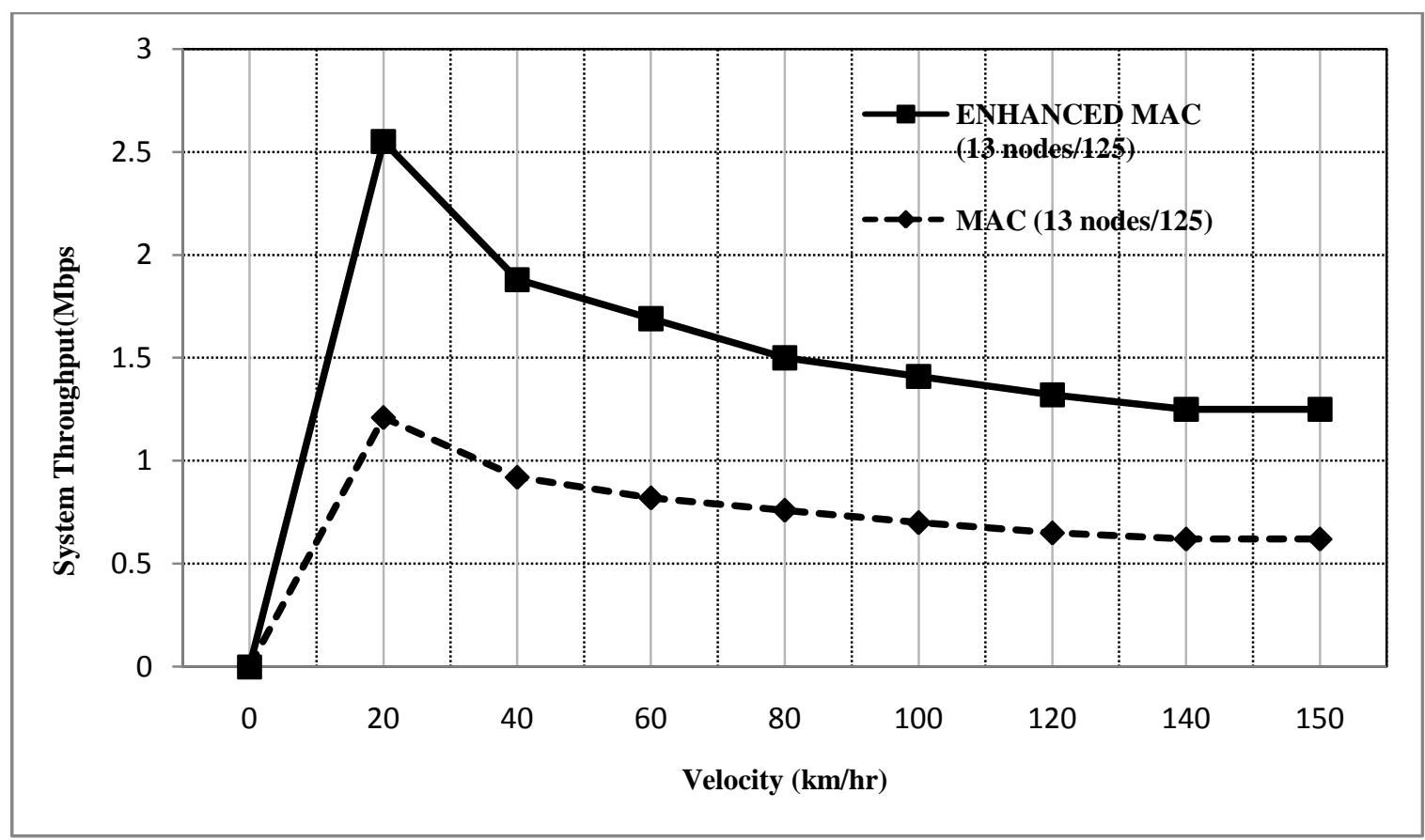

Fig 2: System throughput for different velocities

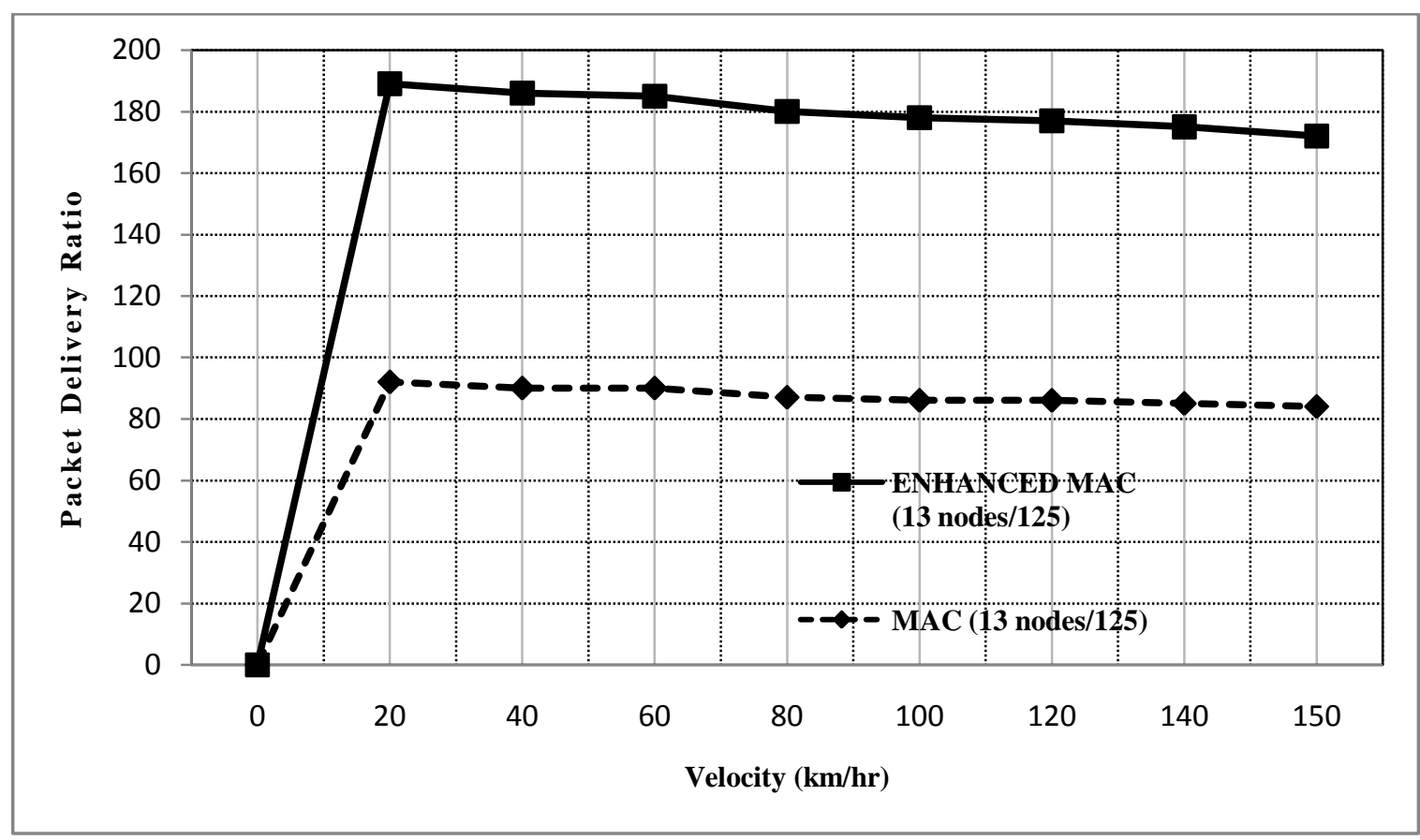

Fig 3: Packet Delivery Ratio for different velocities 


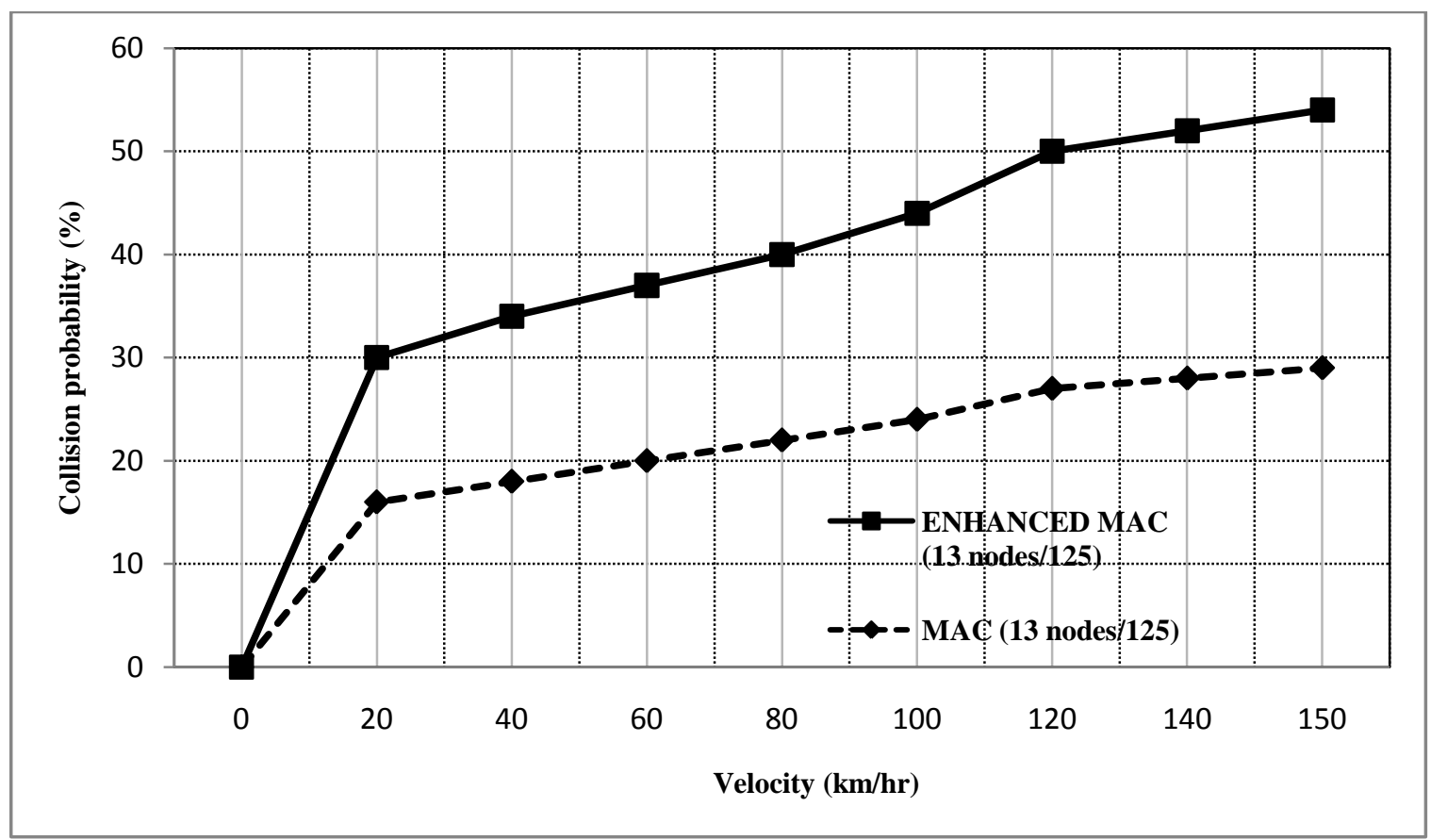

Fig 4: Collision Probability for different velocities

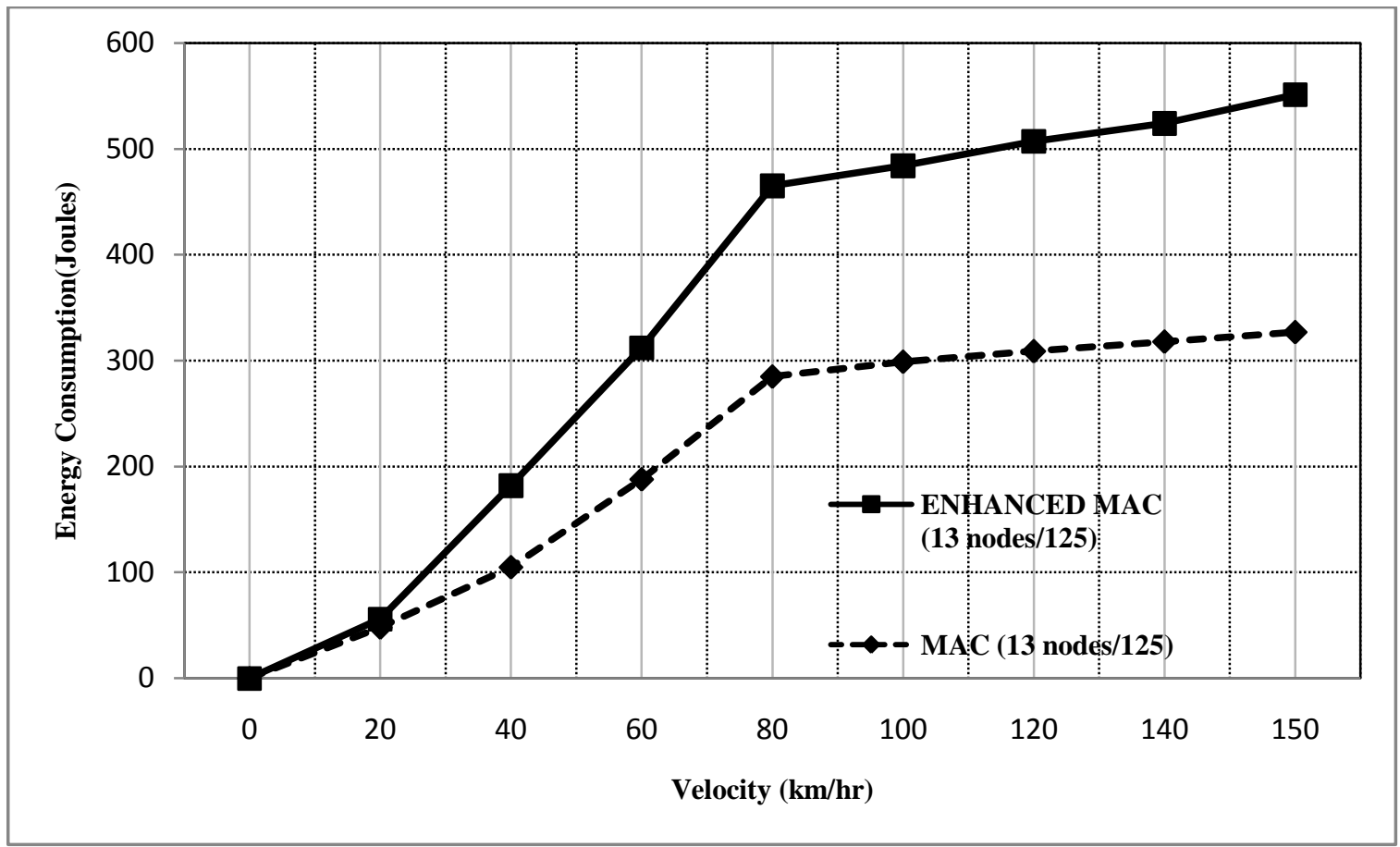

Fig 5: Energy consumption for different velocities 
Table 1. Comparison of System throughput for MAC and

\begin{tabular}{|c|c|c|c|}
\hline \multirow[b]{2}{*}{ Sl.no. } & \multirow[b]{2}{*}{$\begin{array}{l}\text { Velocity } \\
(\mathbf{k m} / \mathbf{h r})\end{array}$} & \multicolumn{2}{|c|}{ System throughput(Mbps) } \\
\hline & & $\begin{array}{c}\text { MAC } \\
(\mathbf{1 3} \\
\text { nodes/125) }\end{array}$ & $\begin{array}{c}\text { ENHANCED } \\
\text { MAC } \\
(13 \\
\text { nodes/125) }\end{array}$ \\
\hline 1 & 0 & 0 & 0 \\
\hline 2 & 20 & 1.21 & 1.34 \\
\hline 3 & 40 & 0.92 & 0.96 \\
\hline 4 & 60 & 0.82 & $\begin{array}{l}0.87 \\
\end{array}$ \\
\hline 5 & 80 & 0.76 & 0.74 \\
\hline 6 & 100 & 0.70 & 0.71 \\
\hline 7 & 120 & 0.65 & 0.67 \\
\hline 8 & 140 & 0.62 & 0.63 \\
\hline 9 & 150 & 0.62 & 0.63 \\
\hline
\end{tabular}

Table 2. Comparison of Packet Delivery ratio for MAC and

\begin{tabular}{|c|c|c|c|}
\hline \multirow{2}{*}{ Sl.no. } & \multirow{2}{*}{ Velocity } & \multicolumn{2}{|c|}{ Packet delivery ratio } \\
\cline { 3 - 4 } & $(\mathbf{k m} / \mathbf{h r})$ & $\begin{array}{c}\text { MAC (13 } \\
\text { nodes/125) }\end{array}$ & $\begin{array}{c}\text { ENHANCED } \\
\text { MAC } \\
(\mathbf{1 3} \\
\text { nodes/125) }\end{array}$ \\
\hline 1 & & 0 & 0 \\
\hline 2 & 20 & 92 & 97 \\
\hline 3 & 40 & 90 & 96 \\
\hline 4 & 60 & 90 & 95 \\
\hline 5 & 80 & 87 & 93 \\
\hline 6 & 100 & 86 & 92 \\
\hline 7 & 120 & 86 & 91 \\
\hline 8 & 140 & 85 & 90 \\
\hline 9 & 150 & 84 & 88 \\
\hline
\end{tabular}

\begin{tabular}{|c|c|c|c|}
\hline \multirow{2}{*}{ Sl.no. } & \multirow{2}{*}{ Velocity } & \multicolumn{2}{|c|}{ Collision probability (\%) } \\
\cline { 3 - 4 } & $(\mathbf{k m} / \mathbf{h r})$ & $\begin{array}{c}\text { MAC } \\
(\mathbf{1 3} \\
\text { nodes/125) }\end{array}$ & $\begin{array}{c}\text { ENHANCED } \\
\text { MAC } \\
(\mathbf{1 3} \\
\text { nodes/125) }\end{array}$ \\
\hline 1 & & 0 & 0 \\
\hline 2 & 20 & 16 & 14 \\
\hline 3 & 40 & 18 & 16 \\
\hline 4 & 60 & 20 & 17 \\
\hline 5 & 80 & 22 & 18 \\
\hline 6 & 100 & 24 & 20 \\
\hline 7 & 120 & 27 & 23 \\
\hline 8 & 140 & 28 & 24 \\
\hline 9 & 150 & 29 & 25 \\
\hline
\end{tabular}

Table 3. Comparison of Collision probability for MAC and enhanced MAC

\begin{tabular}{|c|c|c|c|}
\hline \multirow{2}{*}{ Sl.no. } & \multirow{2}{*}{ Velocity } & \multicolumn{2}{|c|}{ Energy consumption(joules) } \\
\cline { 3 - 4 } & $(\mathbf{k m} / \mathbf{h r})$ & $\begin{array}{c}\text { MAC } \\
\mathbf{( 1 3} \\
\text { nodes/125) }\end{array}$ & $\begin{array}{c}\text { ENHANCED } \\
\text { MAC } \\
(\mathbf{1 3} \\
\text { nodes/125) }\end{array}$ \\
\hline 1 & & 0 & 0 \\
\hline 2 & 20 & 48 & 8 \\
\hline 3 & 40 & 105 & 77 \\
\hline 4 & 60 & 188 & 124 \\
\hline 5 & 80 & 285 & 180 \\
\hline 6 & 100 & 299 & 185 \\
\hline 7 & 120 & 309 & 198 \\
\hline 8 & 140 & 318 & 206 \\
\hline 9 & 150 & 327 & 224 \\
\hline
\end{tabular}

Table 4. Comparison of Energy consumption for MAC and enhanced MAC 


\section{CONCLUSION AND FUTURE WORK}

In this work the internet access in moving vehicles are considered as the main scenario. The impact of mobility is mitigated by adapting the minimum contention window and keeping the back off stage small. To reduce the effect of fading and interference fragmented transmission scheme is applied. By applying fragmented transmission, the loss energy due to unsuccessful transmission can be reduced leading to increased energy efficiency. The trade off between throughput and efficiency can be balanced.

The future research can be done using IEEE 802.11p (WAVE) instead of IEEE 802.11b. IEEE 802.11p uses enhanced distributed channel access (EDCA) in medium access control (MAC) layer using DCF. In VANETs IEEE 802.11p may produce better performance compared to IEEE $802.11 \mathrm{~b}$. So using this standard we can produce higher throughput and efficiency. Instead of FT channel aware back off adjustment(CBT) can be used as the energy efficient transmission scheme which is based on binary exponential back off algorithm(BEB).CBT is based on different sizes of contention window so that which makes system efficient and easier reduction in impact of mobility.

\section{ACKNOWLEDGMENTS}

Authors would like to thank all the researchers who have contributed in this field of research. The comments of anonymous reviewers to improve the quality of this paper are also acknowledged.

\section{REFERENCES}

[1] A. Festag, H. Fußler, H. Hartenstein, A. Sarma, and R. Schmitz 2004, 'FLEETNET: Bringing Car-to-Car Communication into the Real World,' Computer, vol. 4, no. L15, p. 16.

[2] D. Hadaller, S. Keshav, and T. Brecht 2006, 'MV-MAX: Improving Wireless Infrastructure Access for MultiVehicular Communication,' Proc. ACM SIGCOMM Workshop Challenged Networks (CHANTS '06).

[3] F. Cal1', M. Conti, and E. Gregori 2000, 'Dynamic Tuning of the IEEE 802.11 Protocol to Achieve a Theoretical Throughput Limit,' IEEE/ ACM Trans. Networking, vol. 8, no. 6, pp. 785-799.

[4] G. Bianchi 2000, 'Performance Analysis of the IEEE 802. 11 Distributed Coordination Function,' IEEE J. Selected Areas in Comm., vol. 18, no. 3, pp. 535-547.

[5] J. Zhao, T. Arnold, Y. Zhang, and G. Cao 2008, 'Extending Drive-thru Data Access By Vehicle-to-Vehicle Relay,' Proc. Fifth ACM Int'l Workshop VehiculAr Inter-NETworking (VANET'08).

[6] K.-H. Liu, X. Shen, R. Zhang, and L. Cai 2009, 'Performance Analysis of Distributed Reservation Protocol for UWB-Based WPAN', IEEE Trans. Vehicular Technology, vol. 58, no. 2, pp. 902-913.

[7] P. Shankar, T. Nadeem, J. Rosca, and L. Iftode 2008, 'CARS: Context- Aware Rate Selection for Vehicular Networks,' Proc. IEEE Int'l Conf. Network Protocols (ICNP '08).
[8] S. Pack, H. Rutagemwa, X. Shen, J.W. Mark, and K. Park 2008, 'Proxy-Based Wireless Data Access Algorithms in Mobile Hotspots,'IEEE Trans. Vehicular Technology, vol. 57, no. 5, pp. 3165-3177.

[9] Tom H. Luan, Xinhua Ling, and Xuemin (Sherman) Shen, Fellow, IEEE 2012, 'MAC in Motion: Impact of Mobility on the MAC of Drive-Thru Internet', IEEE transactions on mobile computing, Vol. 11, no. 2.

[10] T. Joshi, A. Mukherjee, Y. Yoo, and D.P. Agrawal 2008, 'Airtime Fairness for IEEE 802.11 Multirate Networks,' IEEE Trans. Mobile Computing, vol. 7, no. 4, pp. 513-527.

[11] X. Zhang, J. Kurose, B.N. Levine, D. Towsley, and H. Zhang 2007, 'Study of a Bus-Based Disruption-Tolerant Network: Mobility Modeling and Impact on Routing,' Proc. ACM MobiCom.

[12] Y. Huang, Y. Gao, K. Nahrstedt, and W. He 2009, 'Optimizing File Retrieval in Delay-Tolerant Content Distribution Community,'Proc. IEEE 29th Int'l Conf. Distributed Computing Systems.

[13] Ca Van Phan, Yongsuk Park, Hyo Hyun Choi, Jinsung Cho, and Jeong Geun Kim, 2010 Member, IEEE, 'An EnergyEfficient Transmission Strategy For Wireless Sensor Networks', IEEE Transactions on Consumer Electronics, Vol. 56, No. 2, May.

[14] A. Fu, E. Modiano, and J. N. Tsitsiklis, 2006 "Optimal transmission scheduling over a fading channel with energy and deadline constraints,'IEEE Transactions on Wireless Communications,vol.5,pp.630-641.

[15]A.Ephremides 2002,"Energy concerns in wireless networks2002,"IEEE Transactions on Wireless Communications, vol.9,no.4,pp.48-59,Aug.

[16] A. J. Goldsmith and S. B. Wicker 2002, "Design challenges for energy constrained ad hoc wireless networks,"IEEE Transactions on Wireless Communications, vol. 9, no. 4, pp. 8-27,Aug.

[17] W. Stark, H. Wang 2002, A. Worthen, S. Lafortune, and D. Teneketzis,"Lowenergy wireless communication network design," IEEE Transactions on Wireless Communications, vol. 9, no. 4, pp. 60-72, Aug.

[18] Zhang and K. M. Wasserman 2000, "Energy efficient data communication over fading channels," in Proc. IEEE Wireless Communications and Networking Conf (WCNC'00), Sep., pp. 986-991.

[19] WL Tan, WC Lau, O Yue, TH Hui, 2011, Analytical models and performanceevaluation of drive-thru internet systems. IEEE JSAC.29(1),207-222.

[20] W Alasmary, W Zhuang, 2010, Mobility impact in IEEE 802.11p infrastructure less vehicular networks. Ad Hoc Networks.10(2),222-230 